\title{
Validação de um histórico de enfermagem para indivíduos hospitalizados com doenças infectocontagiosas
}

\author{
Validation of a nursing assessment for hospitalized individuals with infectious diseases
}

Lidiane Lima de Andrade ${ }^{\mathbb{D}}$, Marta Miriam Lopes $\operatorname{Costa}^{2}$ (D), Maria das Graças Melo Fernandes ${ }^{\circledR}$ (D) Suzana de Oliveira Mangueira ${ }^{3}$ (D), Maria Eliane Moreira Freire ${ }^{4}$ (D)

\section{RESUMO}

Analisar a validade dos indicadores empíricos das necessidades humanas básicas contidos em um histórico de enfermagem para indivíduos hospitalizados com doenças infectocontagiosas. Pesquisa metodológica, desenvolvida em duas etapas: análise de conteúdo e validaçáo clínica. Na primeira etapa participaram 37 enfermeiros especialistas e, da segunda, 65 indivíduos internados nas clínicas de doenças infectocontagiosas de dois hospitais universitários. Na validação clínica, foram avaliadas a presença e a ausência dos indicadores empíricos. $\mathrm{Na}$ análise de conteúdo, foram eliminados 14 indicadores empíricos, considerando o critério de relevância. Quanto aos critérios de clareza e precisão, 33 indicadores empíricos tiveram suas definições operacionais reformuladas, com alfa de Cronbach de 0,967 a 0,986. Na validação clínica, foram realizados ajustes para a estruturação final do histórico de enfermagem. Concluiu-se que os indicadores empíricos do histórico de enfermagem representaram adequadamente o foco de interesse e apresentaram excelente consistência interna.

Descritores: Enfermagem; Doenças Transmissíveis; Registros de Enfermagem; Processo de Enfermagem; Estudos de Validação.

\begin{abstract}
To analyze the validity of empirical indicators of basic human needs contained in a nursing assessment for individuals hospitalized with infectious diseases. Methodological study developed in two stages: content analysis and clinical validation. A total of 37 specialist nurses participated in the first stage, and in the second stage there were 65 individuals admitted to the infectious disease clinics of two university hospitals. In the clinical validation, the presence and absence of empirical indicators were evaluated. In the content analysis, 14 empirical indicators were eliminated, considering the relevance criterion. Regarding the criteria of clarity and precision, 33 empirical indicators had their operational definitions reformulated, with Cronbach's alpha from 0.967 to 0.986. In clinical validation, adjustments were made to the final structure of the nursing assessment. It was concluded that the empirical indicators of the nursing assessment adequately represented the focus of interest and showed excellent internal consistency.
\end{abstract}

Descriptors: Nursing; Communicable Diseases; Nursing Records; Nursing Process; Validation Studies.

\footnotetext{
${ }^{1}$ Universidade Federal de Campina Grande - Cuité (PB), Brasil. E-mail:lidiane.lima@ufcg.edu.br

${ }^{2}$ Universidade Federal da Paraíba - João Pessoa (PB), Brasil. E-mails: marthamiryam@hotmail.com; graacafernandes@hotmail.com

${ }^{3}$ Universidade Federal de Pernambuco - Vitória de Santo Antão (PE), Brasil. E-mail: suzanaom@hotmail.com

${ }^{4}$ Hospital Universitário Lauro Wanderley da Universidade Federal da Paraíba - João Pessoa (PB), Brasil. E-mail: enf.elimoreirafreire@gmail.com
}

Como citar este artigo: Andrade LL, Costa MML, Fernandes MGM, Mangueira SO, Freire MEM. Validação de um histórico de enfermagem para indivíduos hospitalizados com doenças infectocontagiosas. Rev. Eletr. Enferm. [Internet]. 2019 [acesso em: ];21:56571. Disponível em: https://doi.org/10.5216/ree.v21.56571. 


\section{INTRODUÇÃO}

A reemergência de enfermidades que se encontravam sob controle, a identificação de novos micro-organismos e a sua disseminação fizeram com que as doenças infectocontagiosas se destacassem entre as morbidades que atingem o ser humano. Essa condição se deve a vários fatores, incluindo o aumento da população global, o envelhecimento, a urbanizaçáo e as mudanças climáticas ${ }^{(1)}$.

Esforços têm sido concentrados para melhorar o cuidado dispensado aos indivíduos com estas afecçóes. O Ministério da Saúde tem criado estratégias, como campanhas de vacinação, implantação da rede de testes rápidos, construção de unidades sentinelas acordadas entre Estados e municípios, elaboração de protocolos de atenção à saúde e repasse financeiro de verbas para a implementação de açóes ${ }^{(2)}$.

Nesse contexto, o profissional de enfermagem, enquanto agente de transformaçáo do processo saúdedoença, apresenta na natureza de sua atuação o dever e a responsabilidade referentes aos serviços e às estruturas de atenção e vigilância em saúde ${ }^{(3)}$.

A visibilidade do cuidado é conferida por meio da documentação da assistência prestada ${ }^{(4)}$. Estima-se que o profissional de enfermagem deve gastar $15 \%$ do seu tempo de trabalho com a documentação, que é a comprovação da quantidade e da qualidade do cuidado(5). Apesar de a documentaçáo ser imprescindível no processo de cuidar em enfermagem, ainda não há padronização universal do registro, existem carência de instrumentos tecnológicos que direcionem a documentação do cuidado e ausência de ferramentas validadas que representem acuradamente o fenômeno clínico de interesse ${ }^{(6,7)}$.

A utilização de instrumentos tecnológicos para registrar as informaçóes relacionadas ao processo de cuidar configura a adoção de um eixo norteador para o cuidado, uma vez que possibilita a avaliaçáo de comportamentos e manifestaçôes clínicas, direciona o raciocínio clínico e a tomada de decisão, operacionalizando, assim, a execução do processo de enfermagem, de maneira a conferir respaldo teórico e eficácia assistencial ${ }^{(8)}$. Nessa perspectiva, é preciso utilizar instrumentos validados, tendo em vista que o processo de validação é um recurso que verifica se um conjunto de itens é abrangente e se representa o assunto em foco ${ }^{(9)}$.

Ademais, reconhecendo a necessidade de aplicar a base de conhecimento na prática, faz-se necessário a utilização de uma teoria de enfermagem. Nesse cenário de estudo, utilizouse a Teoria das Necessidades Humanas Básicas de Horta, por entender que o indivíduo com doenças infectocontagiosas não sofre somente adaptaçóes de ordem fisiológica, devido à exposição a agentes etiológicos, mas também social, emocional e espiritual, principalmente por causa do sentimento de estigma e preconceito.
Diante do exposto, pesquisadores têm ampliado interesse por estudos voltados à construção e/ou à validação de instrumentos para diferentes focos de documentação do cuidado. Ressalta-se a magnitude das doenças infectocontagiosas, visto que, a partir de meados dos anos 60 , com o advento da resistência bacteriana a antimicrobianos e o reaparecimento e a expansão de novas doenças, houve uma polarização epidemiológica nos países em desenvolvimento, com a presença concomitante de elevados índices de morbidade e mortalidade por doenças crônicas não transmissíveis, e da incidência e prevalência de doenças infectocontagiosas, com taxas de mortalidade elevadas em comparação com os países desenvolvidos. No entanto, verifica-se uma incipiência de instrumentos validados para a documentaçáo do processo de enfermagem em indivíduos acometidos por essas afecçôes ${ }^{(10)}$.

Considerando o histórico de enfermagem para indivíduos com doenças infectocontagiosas construído em estudo anterior, ressalta-se a importância de sua validação para aperfeiçoar o conteúdo e representar acuradamente o fenômeno de interesse. Desta forma, o estudo teve como objetivo analisar a validade dos indicadores empíricos das necessidades humanas básicas contidas em um histórico de enfermagem para indivíduos hospitalizados com doenças infectocontagiosas.

\section{MÉTODO}

Trata-se de uma pesquisa metodológica desenvolvida em duas etapas: análise de conteúdo com enfermeiros especialistas e validação clínica com indivíduos hospitalizados com doenças infectocontagiosas.

Considerando que modelos utilizados na validação de diagnósticos de enfermagem ${ }^{(11-13)}$ não foram elaborados para validar outros elementos do cuidado, como histórico, intervençóes e resultados de enfermagem, são necessários ajustes e adaptaçóes para o foco de interesse, aspecto considerado no âmbito desta pesquisa. Levando em consideração as particularidades apresentadas, foram utilizadas algumas etapas metodológicas propostas em um modelo de validação ${ }^{(13)}$, especificamente na análise de conteúdo por especialistas, realizada entre os meses de agosto a novembro de 2015.

Na análise de conteúdo, para a identificação dos especialistas foram realizadas duas buscas na Plataforma Lattes. Na busca 1, utilizou-se como assunto "doenças transmissíveis" e na busca 2, "doenças contagiosas. Ambas as buscas foram feitas nas bases "doutores e demais pesquisadores", com nacionalidade "brasileira” e filtro relativo à área de atuação profissional: grande área "ciências da saúde", área "Enfermagem" e subárea "Enfermagem de doenças contagiosas"; atendendo aos critérios de inclusão: ser enfermeiro com titulação mínima de mestre e desenvolver atividade assistencial em unidade hospitalar 
de doenças infectocontagiosas há, no mínimo, 1 ano; ou ser enfermeiro com titulação mínima de mestre e lecionar disciplina do Curso de Graduação em Enfermagem que versasse sobre o cuidado dispensado a indivíduos acometidos por doenças infectocontagiosas há, no mínimo, 1 ano.

A populaçáo foi definida de acordo com o total de Currículos Lattes selecionados nas referidas buscas, subtraindo-se repetiçóes, o que resultou em 43 especialistas. Utilizaram-se o cálculo para população finita, a confiança de $95 \%$ e o erro amostral de $6 \%$, com o apoio de um programa em linguagem $\mathrm{R}$ (Software $\mathrm{R}$ ) para fazer o cálculo, que, depois de executado, chegou a uma amostra de 37 indivíduos.

Para análise de conteúdo foi construído um instrumento de coleta de dados dividido em duas partes. A primeira continha dados de caracterização dos especialistas, e a segunda, indicadores empíricos da teoria das necessidades humanas básicas ${ }^{(14,15)}$, distribuídos em: necessidades psicobiológicas oxigenação (19), hidratação (3), nutrição (28), eliminação (25), sono e repouso (6), atividade física (11), sexualidade e reprodução (5), segurança física e do meio ambiente (13), cuidado corporal (17), integridade física (28), regulação vascular (17), regulação térmica (4), regulação neurológica (18), sensopercepção (6), terapêutica e prevenção (9); necessidades psicossociais - comunicação (8), gregária (4), recreação e lazer (1), segurança emocional (16); autoestima, autoconfiança e autorrespeito (9), educação para a saúde e aprendizagem (6); necessidades psicoespirituais — religiosidade e espiritualidade (5), contabilizando 258 indicadores empíricos.

Solicitou-se aos especialistas a análise dos indicadores empíricos das necessidades humanas básicas e suas respectivas definiçóes operacionais, com base nos critérios de relevância, clareza e precisão. Relevância referiu-se à pertinência e à possibilidade de o indicador e a definição serem importantes; por clareza, tomou-se a capacidade de o indicador e a definição serem inteligíveis, com frases curtas; precisão foi compreendida como a capacidade do indicador e a definição de ter uma posição delimitada e ser distinto dos demais ${ }^{(16)}$. Para cada critério analisado, foram apresentadas as seguintes opçóes de respostas: zero, se inadequado (inapropriado, quanto ao critério em análise); 1, se parcialmente adequado (incompleto e insuficiente, quanto ao critério em análise); e 2, se adequado (apropriado e conveniente, quanto ao critério em análise). Caso o especialista considerasse algum indicador como zero ou 1, era solicitado que utilizasse o espaço indicado para justificativa e sugestóes de modificação.

$\mathrm{Na}$ análise foram utilizados os testes de proporção, considerando a concordância mínima de $80 \%$, e o teste binominal. Para a realização do teste binomial, a escala com três pontos foi recodificada em dois pontos, categorizados como inadequados valores iguais a zero e um, e considerados adequados valores iguais a dois, necessários devido à natureza dicotômica do teste.
No critério de relevância, foram eliminados os indicadores empíricos que não atingiram a concordância mínima de $80 \%$ e apresentaram valor-p acima de 0,05 , e os que não atingiram a concordância mínima de $80 \%$ e apresentaram valor-p abaixo de 0,05 , considerando a bilateralidade do teste binomial. Quanto aos critérios de clareza e precisão, foram modificadas as definiçóes operacionais dos indicadores empíricos que não atingiram a concordância mínima de $80 \%$ e apresentaram valor-p acima de 0,05 , e os que não atingiram a concordância mínima de $80 \%$ e apresentaram valor-p abaixo de 0,05 , considerando a bilateralidade do teste binomial. As modificaçóes ocorreram de acordo com o consolidado nas sugestóes apresentadas pelos especialistas. Para mensurar a fidedignidade do teste, utilizou-se o alfa de Cronbach, e considerou-se aceitável um escore de confiabilidade entre os valores de 0,7 a $1^{(17)}$.

No tocante à validação clínica, tendo em vista a inexistência de referenciais metodológicos pertinentes à validação do histórico de enfermagem, objeto desta análise, adotaram-se critérios específicos à seleção dos indivíduos hospitalizados para determinar a amostra e proceder ao tratamento estatístico dos dados.

A etapa de validação clínica foi desenvolvida em unidades de internação de doenças infectocontagiosas de dois hospitais universitários do Estado da Paraíba, na Região Nordeste do Brasil. Foram incluídos os indivíduos hospitalizados no período da coleta dos dados, compreendido entre os meses de fevereiro a abril de 2016, e excluídos os menores de 18 anos e os indivíduos que não apresentavam doenças infectocontagiosas.

A população foi delimitada com base em um levantamento das admissóes nas unidades de internação lócus da pesquisa. Assim, foi definida a média de internação nos meses de fevereiro a abril de 2014 e 2015. Foram hospitalizados 104 indivíduos. Utilizaram-se cálculo para população finita, confiança de $95 \%$ e erro amostral de $7,5 \%$, e utilizou-se um programa em linguagem $\mathrm{R}$ (Software $\mathrm{R}$ ) para fazer o cálculo que, depois de executado, chegou a uma amostra de 65.

Na validação clínica, foi utilizado o instrumento analisado na etapa anterior pelos enfermeiros especialistas, composto pelos indicadores empíricos das necessidades humanas básicas. Estes indicadores foram avaliados com base nas técnicas de anamnese e de exame físico, e julgados quanto à presença ou à ausência, quando aplicados aos indivíduos hospitalizados com doenças infectocontagiosas.

A coleta de dados foi realizada pela pesquisadora e contou com o auxílio de colaboradores. Antes de aplicar o instrumento, os colaboradores participaram de um treinamento, em que foram abordados aspectos como anamnese e exame físico da população estudada, pautandose nas definiçóes operacionais dos indicadores empíricos presentes no histórico de enfermagem. 
$\mathrm{Na}$ validação clínica foram avaliadas a presença e a ausência dos indicadores empíricos das necessidades humanas básicas, o que respaldou os ajustes e a apresentação final do histórico de enfermagem para indivíduos hospitalizados com doenças infectocontagiosas.

A pesquisa foi aprovada pelo Comitê de Ética em Pesquisa da instituição vinculada ao estudo, sob protocolo de aprovaçáo no 1.663 .005 .

\section{RESULTADOS}

$\mathrm{Na}$ etapa de análise de conteúdo, verificou-se que, dentre os enfermeiros especialistas, a maioria era do sexo feminino (94,6\%), com procedência da Região Nordeste (78,3\%), apresentando média de idade de 41,08 $( \pm 9,54)$ anos, título de mestrado (62,2\%), e experiência em ensino na área de doenças infectocontagiosas $(58,7 \%)$, com média de 11,22 $( \pm 10,55)$ anos de atuação.

No tocante à avaliação dos indicadores empíricos por enfermeiros especialistas, 14 foram eliminados, conforme o critério de relevância, e 33 tiveram suas definiçóes operacionais modificadas, de acordo com os critérios de clareza e precisão (Tabela 1 ).

Para comprovar a consistência interna dos indicadores empíricos, segundo os critérios mencionados, foi verificado o alfa de Cronbach, conforme demonstra a Tabela 2.

Quanto à validação clínica com indivíduos hospitalizados, constatou-se que a maioria era do sexo masculino $(53,8 \%)$, com média de 44,5 $( \pm 16,42)$ anos, solteiros $(41,5 \%)$, desenvolvia como atividade de ocupação do lar $(21,5 \%)$, residia no interior do Estado (50,8\%), tinha como principal diagnóstico médico HIV/AIDS (30,8\%) e apresentava como principais motivos de internação: febre $(17,1 \%)$, cefaleia $(7,7 \%)$, vômito $(6,1 \%)$; tosse, diarreia e mialgia, com $5,5 \%$, cada.

O levantamento dos indicadores empíricos ausentes e presentes embasou ajustes na composição do histórico de enfermagem para indivíduos hospitalizados com doenças infectocontagiosas, conforme Quadro 1. Quanto aos ausentes, constatou-se que 27 indicadores empíricos não foram identificados: murmúrios vesiculares ausentes, uso de traqueostomia, ascite, anasarca, uso de sonda nasogástrica fechada, anúria, oligúria, colúria, enterorragia, melena, piúria, retenção urinária, uso de sonda nasogástrica aberta, apneia do sono, pediculose, deiscência, evisceração, cianose, hematomas, hemorragia, decorticação, inconsciente, opistótono, pupilas anisocóricas, riso sardônico, trismo e efeitos adversos.

\section{DISCUSSÃO}

A partir da análise de conteúdo, constatou-se uma expressividade na eliminação de indicadores empíricos da necessidade psicobiológica de oxigenação, como: batimentos das aletas nasais, expectoração fluida e gemência. $\mathrm{O}$ primeiro foi considerado redundante, por ser uma manifestação clínica da dispneia, termo já contemplado. A expectoração fluida foi julgada como não específica de indivíduos com doenças infectocontagiosas, tendo em vista que indivíduos com infecção pulmonar geralmente apresentam expectoração purulenta ${ }^{(18)}$. Já a gemência por ser um sinal muito comum em recém-nascidos e lactentes, visto que o histórico de enfermagem é destinado a maiores de 18 anos.

Dentre as doenças infectocontagiosas, ressaltam-se como necessidade de oxigenação, na tuberculose pulmonar, a tosse com ou sem expectoração, a hemoptise e a dispneia ${ }^{(19)}$. Esses dados foram atestados em um estudo retrospectivo desenvolvido com indivíduos com as comorbidades HIV/ AIDS e tuberculose, apontando que, destes, 38,3\% apresentavam tosse crônica ${ }^{(20)}$.

Destaca-se a grande quantidade de alteraçôes nas definiçôes operacionais dos indicadores empíricos da necessidade de nutrição, principalmente quanto à utilização dos métodos propedêuticos para a avaliação abdominal.

Assim, no abdômen distendido foi acrescentada, além da inspeção, o método propedêutico de percussão, tendo em vista a avaliação do acúmulo de gases, líquidos e sólidos na cavidade abdominal; no abdômen flácido, adicionada a causa, por entender que essa é resultante do enfraquecimento muscular, que diminui sua firmeza e elasticidade.

A definiçáo do abdômen globoso foi considerada confusa com o abdômen volumoso, e, dessa forma, consideraram-se o primeiro como o aumento do diâmetro anteroposterior do abdômen, e o segundo como aumento de seu diâmetro transversal.

Também foi modificada a definição de abdômen maciço, por não ser considerado na Semiotécnica como um tipo abdominal, mas como um som abdominal obtido pela percussão de vísceras maciças.

A flacidez abdominal pode ser percebida em pessoas acometidas pelo HIV/AIDS como efeito adverso da terapia antiretroviral, devido à lipodistrofia, classificada em lipohipertrofia, quando há acúmulo de gordura. Geralmente, a área do corpo mais acometida pela lipo-hipertrofia é a região abdominal $^{(21)}$. Esse achado foi observado em estudo que relacionou os maiores índices de lipo-hipertrofia em pacientes com excesso de peso, o que é possivelmente explicado pelo maior acúmulo de gordura na região abdominal(22).

Os abdomens globoso e o volumoso são comuns em indivíduos obesos, com ascite, com hepatomegalia e com esplenomegalia. Estudo demonstrou que essas manifestaçóes clínicas podem ser evidenciadas em indivíduos com tuberculose, doenças hepáticas, filariose e alcoolismo ${ }^{(23)}$.

Nas necessidades psicossociais, observou-se a eliminação de indicadores empíricos da necessidade de autoestima, autoconfiança e autorrespeito, como: desejo de conquista e 
Tabela 1. Distribuição dos indicadores empíricos das necessidades humanas básicas conforme análise dos especialistas. João Pessoa, PB, Brasil, 2016.

\begin{tabular}{l|c|c|c|c|c|c|} 
Indicadores empíricos das & \multicolumn{2}{|c|}{ Relevância } & \multicolumn{2}{|c|}{ Clareza } & \multicolumn{2}{|c}{ Precisão } \\
\cline { 2 - 7 } necessidades humanas básicas & $\mathrm{n}(\%)$ & valor-p & $\mathrm{n}(\%)$ & valor-p & $\mathrm{N}(\%)$ & valor-p
\end{tabular}

Necessidades psicobiológicas

Oxigenação

\begin{tabular}{|c|c|c|c|c|c|c|}
\hline Batimento das aletas nasais & $28(75,7)$ & 0,511 & - & - & - & - \\
\hline Dispneia & $32(86,5)$ & 0,324 & $18(48,6)$ & 0,000 & $19(51,4)$ & 0,000 \\
\hline Expectoração espessa & $33(89,2)$ & 0,162 & $29(78,4)$ & 0,805 & $27(73,0)$ & 0,285 \\
\hline Expectoração fluida & $29(78,4)$ & 0,805 & - & - & - & - \\
\hline Gemência & $26(70,3)$ & 0,139 & - & - & - & - \\
\hline Murmúrios vesiculares ausentes & $33(89,2)$ & 0,162 & $24(64,9)$ & 0,021 & $30(81,1)$ & 0,869 \\
\hline \multicolumn{7}{|l|}{ Nutrição } \\
\hline Abdômen distendido & $34(91,9)$ & 0,071 & $21(56,8)$ & 0,000 & $25(67,6)$ & 0,059 \\
\hline Abdômen flácido & $30(81,1)$ & 0,869 & $23(62,2)$ & 0,007 & $25(67,6)$ & 0,059 \\
\hline Abdômen globoso & $33(89,2)$ & 0,162 & $27(73,0)$ & 0,285 & $27(73,0)$ & 0,285 \\
\hline Abdômen maciço & $33(89,2)$ & 0,162 & $30(81,1)$ & 0,869 & $29(78,4)$ & 0,805 \\
\hline Abdômen volumoso & $30(81,1)$ & 0,869 & $27(73,0)$ & 0,285 & $29(78,4)$ & 0,805 \\
\hline Aceitação parcial da dieta & $35(94,6)$ & 0,026 & $27(73,0)$ & 0,285 & $26(70,3)$ & 0,139 \\
\hline Aceitação total da dieta & $33(89,2)$ & 0,162 & $29(78,4)$ & 0,805 & $29(78,4)$ & 0,805 \\
\hline Anorexia & $37(100,0)$ & 0,002 & $28(75,7)$ & 0,511 & $28(75,7)$ & 0,511 \\
\hline Apetite diminuído & $34(91,9)$ & 0,071 & $31(83,8)$ & 0,565 & $28(75,7)$ & 0,511 \\
\hline Esplenomegalia & $36(97,3)$ & 0,009 & $29(78,4)$ & 0,805 & $31(83,8)$ & 0,565 \\
\hline Não aceita dieta oral & $34(91,9)$ & 0,071 & $29(78,4)$ & 0,805 & $28(75,7)$ & 0,511 \\
\hline Preferências alimentares & $28(75,7)$ & 0,511 & - & - & - & - \\
\hline Singulto & $29(78,4)$ & 0,805 & - & - & - & - \\
\hline \multicolumn{7}{|l|}{ Eliminação } \\
\hline Aspecto das fezes (odor) & $33(89,2)$ & 0,162 & $28(75,7)$ & 0,511 & $30(81,1)$ & 0,869 \\
\hline Flatulência & $32(86,5)$ & 0,324 & $28(75,7)$ & 0,511 & $31(83,8)$ & 0,565 \\
\hline Incontinência urinária & $34(91,9)$ & 0,071 & $25(67,6)$ & 0,059 & $32(86,5)$ & 0,324 \\
\hline Retenção urinária & $36(97,3)$ & 0,009 & $28(75,7)$ & 0,511 & $33(89,2)$ & 0,162 \\
\hline Uso de sonda nasogástrica aberta & $34(91,9)$ & 0,071 & $29(78,4)$ & 0,805 & $31(83,8)$ & 0,565 \\
\hline \multicolumn{7}{|l|}{ Sono e repouso } \\
\hline Sono satisfatório & $32(86,5)$ & 0,324 & $29(78,4)$ & 0,805 & $33(89,2)$ & 0,162 \\
\hline \multicolumn{7}{|l|}{ Atividade física } \\
\hline Atrofia muscular & $34(91,9)$ & 0,071 & $27(73,0)$ & 0,285 & $27(73,0)$ & 0,285 \\
\hline \multicolumn{7}{|l|}{ Sexualidade e reprodução } \\
\hline $\begin{array}{l}\text { Presença de doenças } \\
\text { sexualmente transmissíveis }\end{array}$ & $34(91,9)$ & 0,071 & $28(75,7)$ & 0,511 & $24(64,9)$ & 0,021 \\
\hline \multicolumn{7}{|l|}{ Cuidado corporal } \\
\hline Caspas & $27(73,0)$ & 0,285 & - & - & - & - \\
\hline Uso de aparelho ortodôntico & $25(67,6)$ & 0,059 & - & - & - & - \\
\hline
\end{tabular}


Tabela l. Continuação.

\begin{tabular}{|c|c|c|c|c|c|c|}
\hline \multirow{2}{*}{$\begin{array}{l}\text { Indicadores empíricos das } \\
\text { necessidades humanas básicas }\end{array}$} & \multicolumn{2}{|c|}{ Relevância } & \multicolumn{2}{|c|}{ Clareza } & \multicolumn{2}{|c|}{ Precisão } \\
\hline & $n(\%)$ & valor-p & $n(\%)$ & valor-p & $N(\%)$ & valor-p \\
\hline \multicolumn{7}{|l|}{ Integridade física } \\
\hline Exantema & $36(97,3)$ & 0,009 & $29(78,4)$ & 0,805 & $31(83,8)$ & 0,565 \\
\hline \multicolumn{7}{|l|}{ Regulação vascular } \\
\hline Prova do laço negativa & $35(94,6)$ & 0,026 & $29(78,4)$ & 0,805 & $33(89,2)$ & 0,162 \\
\hline \multicolumn{7}{|l|}{ Regulação térmica } \\
\hline Piloereção & $27(73,0)$ & 0,285 & - & - & - & - \\
\hline \multicolumn{7}{|l|}{ Sensopercepção } \\
\hline $\begin{array}{l}\text { Apresenta expressões corporais } \\
\text { relacionadas à dor }\end{array}$ & $34(91,9)$ & 0,071 & $29(78,4)$ & 0,805 & $29(78,4)$ & 0,805 \\
\hline Fatores que aumentam a dor & $34(91,9)$ & 0,071 & $29(78,4)$ & 0,805 & $31(83,8)$ & 0,565 \\
\hline \multicolumn{7}{|l|}{ Terapêutica e prevenção } \\
\hline Cartão de vacina atualizado & $36(97,3)$ & 0,009 & $27(73,0)$ & 0,285 & $30(81,1)$ & 0,869 \\
\hline Efeitos colaterais & $36(97,3)$ & 0,009 & $29(78,4)$ & 0,805 & $30(81,1)$ & 0,869 \\
\hline \multicolumn{7}{|l|}{ Segurança física e do meio ambiente } \\
\hline Água tratada & $34(91,9)$ & 0,071 & $26(70,3)$ & 0,139 & $30(81,1)$ & 0,869 \\
\hline Destino do lixo & $34(91,9)$ & 0,071 & $32(86,5)$ & 0,324 & $29(78,4)$ & 0,805 \\
\hline $\begin{array}{l}\text { Hábito de higienizar os } \\
\text { alimentos }\end{array}$ & $35(94,6)$ & 0,026 & $34(91,9)$ & 0,071 & $29(78,4)$ & 0,805 \\
\hline \multicolumn{7}{|l|}{ Necessidades psicossociais } \\
\hline \multicolumn{7}{|l|}{ Comunicação } \\
\hline Gagueira & $29(78,4)$ & 0,805 & - & - & - & - \\
\hline \multicolumn{7}{|l|}{ Recreação e lazer } \\
\hline Ocupação do tempo livre & $29(78,4)$ & 0,805 & - & - & - & - \\
\hline \multicolumn{7}{|l|}{ Segurança emocional } \\
\hline Frustração & $29(78,4)$ & 0,805 & - & - & - & - \\
\hline \multicolumn{7}{|c|}{ Autoestima, autoconfiança e autorrespeito } \\
\hline $\begin{array}{l}\text { Atitudes relacionadas } \\
\text { ao próprio corpo }\end{array}$ & $32(86,5)$ & 0,324 & $30(81,1)$ & 0,869 & $28(75,7)$ & 0,511 \\
\hline Desejo de conquista e vitória & $29(78,4)$ & 0,805 & - & - & - & - \\
\hline $\begin{array}{l}\text { Reconhece que é merecedor } \\
\text { de amor e felicidade }\end{array}$ & $28(75,7)$ & 0,511 & - & - & - & - \\
\hline Tem medo de expor suas ideias & $28(75,7)$ & 0,511 & - & - & - & - \\
\hline \multicolumn{7}{|l|}{ Necessidades psicoespirituais } \\
\hline \multicolumn{7}{|l|}{ Religiosidade e espiritualidade } \\
\hline Religião & $34(91,9)$ & 0,071 & $32(86,5)$ & 0,324 & $29(78,4)$ & 0,805 \\
\hline
\end{tabular}

Tabela 2. Análise da fidedignidade dos indicadores empíricos. João Pessoa, Paraíba, Brasil, 2016.

\begin{tabular}{l|c|c|c}
\multirow{2}{*}{ Critérios } & Indicadores empíricos & Alfa de Cronbach & Intervalo a 95\% \\
\cline { 2 - 4 } & 258 & 0,986 & 0,979 a 0,992 \\
\hline \multirow{2}{*}{ Clarezância } & 244 & 0,986 & 0,979 a 0,992 \\
\hline Precisão & 244 & 0,967 & 0,951 a 0,981 \\
\hline
\end{tabular}


Quadro l. Histórico de enfermagem para indivíduos hospitalizados com doenças infectocontagiosas.

HISTÓRICO DE ENFERMAGEM PARA INDIVÍDUOS HOSPITALIZADOS COM DOENÇAS INFECTOCONTAGIOSAS DADOS DE IDENTIFICAÇÃO

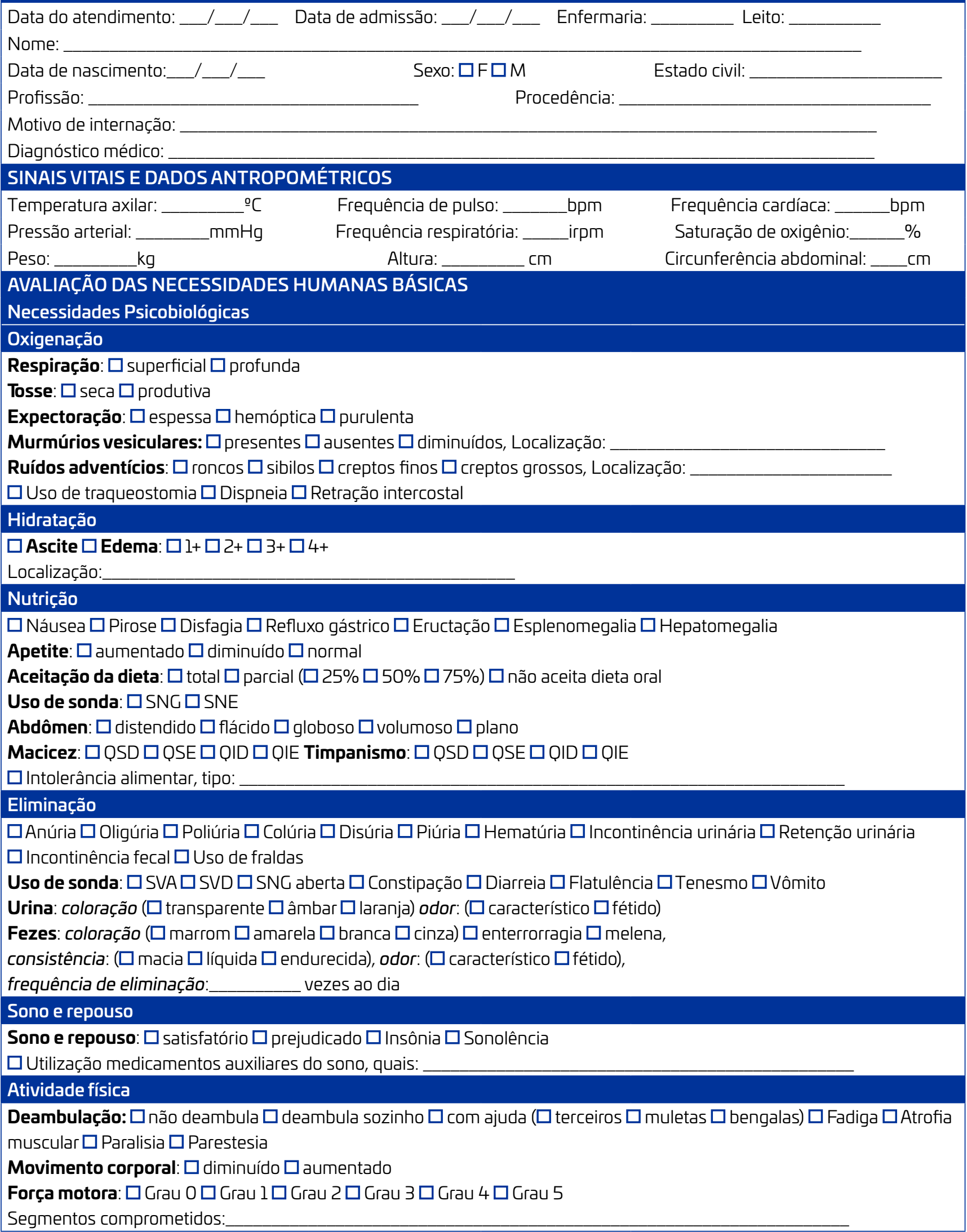


Quadro l. Continuação.

Sexualidade e reprodução

$\square$ Vida sexual ativa

Relacionamento: $\square$ homossexual $\square$ bissexual $\square$ heterossexual

Utilização de métodos contraceptivos: $\square$ camisinha $\square$ pílula $\square$ outros, quais:

Cuidado corporal

Higiene corporal: $\square$ preservada $\square$ prejudicada

Higiene íntima: $\square$ preservada $\square$ prejudicada

Higiene oral: $\square$ preservada $\square$ prejudicada $\square$ Cáries $\square$ Falhas dentárias $\square$ Uso de prótese dentária $\square$ Halitose

Dependência do autocuidado: $\square$ total $\square$ parcial $\square$ (banhar-se $\square$ arrumar-se $\square$ vestir-se $\square$ alimentar-se)

Seborreia Alopecia, localização:

Integridade física

Turgor da pele/elasticidade da pele: $\square$ preservada $\square$ diminuída $\square$ Prurido $\square$ Icterícia $\square$ Fístula

Lesões, localização:

Exantema: $\square$ (Petéquias $\square$ Mácula $\square$ Pápula $\square$ Vesícula $\square$ Pústula) $\square$ Crosta

Manchas: $\square$ hipercrômicas $\square$ hipocrômicas $\square$ hiperemiadas

Sensibilidade das lesões: $\square$ dolorosa $\square$ tátil $\square$ térmica

Lesão por pressão: $\square$ estágio $1 \square$ estágio $2 \square$ estágio $3 \square$ estágio $4 \square$ não classificável $\square$ tissular profunda

Avaliação das feridas: leito ( $\square$ granulação $\square$ fibrina $\square$ necrose de coagulação $\square$ necrose de liquefação)

bordas ( $\square$ distintas $\square$ indistintas $\square$ maceradas) fase de cicatrização ( $\square$ inflamatória $\square$ proliferativa $\square$ maturação)

exsudato - coloração ( $\square$ seroso $\square$ hemático $\square$ serohemático $\square$ purulento) odor ( $\square$ característico $\square$ fétido $\square$ pútrido)

quantidade ( $\square$ pouco $\square$ moderado $\square$ abundante)

Tratamento: limpeza: e

cobertura:

Regulação vascular

Pulso: $\square$ cheio $\square$ filiforme

Perfusão periférica: $\square$ preservada $\square$ diminuída

Ritmo cardíaco: $\square$ regular $\square$ irregular

Bulhas cardíacas: $\square$ normofonéticas $\square$ hipofonéticas $\square$ hiperfonéticas

(focos auscultados: $\square$ pulmonar $\square$ aórtico $\square$ tricúspide mitral) $\square$ Equimose $\square$ Hematomas $\square$ Cianose

Prova do laço: $\square$ positiva $\square$ negativa

Perdas sanguíneas, localização:

Regulação térmica

$\square$ Pele fria $\square$ Calafrios $\square$ Sudorese ( $\square$ manhã $\square$ tarde $\square$ noite)

Regulação neurológica

Nível de consciência: pontuação dos escores (ocular [ ] verbal [ ] motora [ ])

$\square$ Desorientação $\square$ Agitação $\square$ Crise convulsiva $\square$ Decorticação

Avaliação pupilar: $\square$ pupilas fotorreagentes $\square$ isocóricas $\square$ anisocóricas

Alteração nos reflexos: $\square$ braquiorradial $\square$ bicipital $\square$ tricipital $\square$ patelar Graduações: $\square 0 \square$ l+ $\square 2+\square 3+\square$ 4+

$\square$ Fotofobia $\square$ Rigidez de nuca $\square$ Sinal de Brudzinski $\square$ Sinal de Kernig $\square$ Riso sardônico $\square$ Trismo $\square$ Opistótono

Sensopercepção

$\square$ Dor, localização:

Frequência: $\square$ l a 5 vezes por dia $\square$ mais de 5 vezes $\square$ contínua

Intensidade: $\square$ leve $\square$ moderada $\square$ intensa

Expressões corporais relacionadas à dor: $\square$ sobrancelhas franzidas $\square$ proteção à área $\square$ inquietação $\square$ choro

Fatores que aliviam: $\square$ medidas farmacológicas $\square$ medidas não farmacológicas, quais:

Fatores que aumentam: $\square$ movimentação $\square$ deambulação $\square$ frio $\square$ manipulação durante a realização do curativo 
Quadro l. Continuação.

Segurança física e do meio ambiente

Uso de água tratada: $\square$ filtrada $\square$ fervida $\square$ clorada $\square$ mineral $\square$ proveniente da companhia de abastecimento

Condições de moradia: $\square$ casa de alvenaria $\square$ casa de taipa $\square$ rua calçada $\square$ rua sem calçamento

Destino do lixo: $\square$ coleta pela prefeitura $\square$ aterro sanitário $\square$ queima $\square$ a céu aberto

$\square$ Hábito de higienizar as mãos $\square$ Hábito de higienizar os alimentos $\square$ Necessidade utilizar máscaras para transporte

$\square$ Criação de animais, quais:

$\square$ Dependente químico, tipo de dependência:

$\square$ Etilista, desde quando:

frequência de consumo ao dia:

口Tabagista, desde quando: frequência de consumo ao dia

Terapêutica e prevenção

$\square$ Reações alérgicas $\square$ Efeitos colaterais $\square$ Efeitos adversos, descrição:

口Doenças crônicas, descrição: $\square$ Sinais flogísticos de inserção dos cateteres

$\square$ Situações que interferem no tratamento, descrição:

Necessidades Psicossociais

Comunicação

$\square$ Afasia $\square$ Disartria

Uso de linguagem: $\square$ verbal $\square$ não verbal $\square$ Respostas desconexas

Interação com as pessoas: $\square$ pouco comunicativo $\square$ comunicativo $\square$ Habilidades para escrever

Gregária

Quem reside na casa/apto: $\square$ mora sozinho $\square$ familiares $\square$ amigos

Número de pessoas com quem reside: [ ] ]

$\square$ Desejo de participar de atividades cotidianas

Quem mais auxilia no tratamento: $\square$ familiares $\square$ amigos

Segurança emocional

$\square$ Agressividade $\square$ Angústia $\square$ Ansiedade $\square$ Apatia $\square$ Carência afetiva $\square$ Choro $\square$ Desejo de se isolar $\square$ Depressão

$\square$ Desejo de morrer $\square$ Inconstância de humor $\square$ Irritabilidade $\square$ Sentimento de estigma e preconceito $\square$ risteza

$\square$ Medo/fobia, descrição:

Autoestima, autoconfiança e autorrespeito

$\square$ Falta de autoconfiança $\square$ Atitudes relacionadas à autoimagem:

Percepções sobre a autoimagem: Fontes de motivação:

Atributos positivos em si mesmo:

Educação para a saúde e aprendizagem

Nível de escolaridade: $\square$ não alfabetizado $\square$ ensino fundamental $\square$ ensino médio $\square$ graduação $\square$ pós-graduação

Experiências anteriores: $\square$ doenças $\square$ cirurgias $\square$ tratamentos, descrição:

Conhecimento sobre problema de saúde: $\square$ nenhum $\square$ diagnóstico $\square$ como adquiriu $\square$ forma de transmissão

$\square$ sinais e sintomas $\square$ ação dos medicamentos

O que a família sabe sobre o problema de saúde: $\square$ nada $\square$ diagnóstico $\square$ como adquiriu $\square$ forma de transmissão

$\square$ sinais e sintomas $\square$ ação dos medicamentos

Necessidades Psicoespirituais

Religiosidade e espiritualidade

Crenças: $\square$ espirituais $\square$ religiosas $\square$ culturais $\square$ Necessidade da presença de um líder espiritual

Religião: $\square$ católica $\square$ evangélica $\square$ adventista $\square$ testemunha de jeová $\square$ candomblé $\square$ Outras

QSD: quadrante superior direito; QSE: quadrante superior esquerdo; QIE: Quadrante inferior esquerdo; QID: Quadrante inferior direito. 
vitória, e reconhece que é merecedor de amor e felicidade. Esses se referem a atributos positivos da necessidade, que nem sempre, são encontrados no indivíduo hospitalizado, devido à mudança na rotina e ao distanciamento familiar, que resulta em fragilidade, tendo em vista que o processo de hospitalização gera uma alteração biopsicossocial e espiritual do ser humano ${ }^{(24)}$.

Nas necessidades psicoespirituais, foi alterada a definição operacional do indicador empírico religião, pois estava restrita à necessidade de pertencer a uma entidade religiosa. Desta maneira, acredita-se que a relação entre religiosidade e indivíduos diagnosticados com doenças sem possibilidade de cura configura-se importante, porquanto requer a agregação de diferentes parâmetros culturais, conceitos morais e ideais específicas que atribuem sentido à existência humana ${ }^{(25)}$.

$\mathrm{Na}$ validação clínica, infere-se que a não ocorrência de alguns indicadores empíricos deve-se ao fato de que determinadas manifestações clínicas são específicas de doenças que não foram identificadas nos participantes do estudo, como, por exemplo, as hepatites virais e o tétano acidental.

Os limites do estudo estiveram relacionados à especificidade das manifestaçóes clínicas dos indivíduos hospitalizados no período de coleta de dados. Esse fato é atribuído ao recorte temporal estabelecido, que compreendia meses chuvosos, visto que a sazonalidade exerce influência no perfil de internaçóes das doenças infectocontagiosas. Assim, recomenda-se que sejam elaboradas outras validaçóes clínicas utilizando-se recortes temporais com estiagem pluviométrica, e validação clínica em outros contextos e locais, devido à endemicidade das doenças infectocontagiosas.

\section{CONCLUSÃO}

Os indicadores empíricos do histórico de enfermagem para indivíduos hospitalizados com doenças infectocontagiosas representaram adequadamente o foco de interesse. Ademais, houve excelente consistência interna, o que aponta que, com esses valores de fidedignidade, tem-se confiança maior na apresentação final do histórico de enfermagem.

Mesmo reconhecendo o avanço da era digital na Enfermagem, optou-se pela construção de um histórico de enfermagem em check list. Essa escolha é justificada pela incipiência de instrumentos validados para a documentação de enfermagem na área de doenças infectocontagiosas na literatura nacional. Ademais, os resultados dessa pesquisa poderão embasar a construção de tecnologias de informação, por meio do desenvolvimento de softwares.

Convém destacar a relevância da validação de instrumentos que respaldem a documentação de Enfermagem, sobretudo ferramentas para a avaliação inicial, como o histórico de enfermagem, que subsidia a implementação das demais etapas do processo de enfermagem. Além disso, a utilização de recursos validados confere a um conjunto de itens a representatividade do fenômeno de interesse. Dessa forma, os achados dessa pesquisa fortalecerão a prática clínica, o ensino e a comunidade científica da área de enfermagem em doenças infectocontagiosas.

\section{REFERÊNCIAS}

1. Bloom DE, Black S, Rappuoli R. Emerging infectious diseases: A proactive approach. Proc Natl Acad Sci U S A [Internet]. 2017 [acessado em 19 dez. 2018];114(16):4055-9. Disponível em: https:// www.pnas.org/content/114/16/4055. https://doi. org/10.1073/pnas.1701410114.

2. Brasil. Ministério da Saúde. Secretaria de Vigilância em Saúde. Monitoramento dos casos de dengue, febre de chikungunya e febre pelo vírus Zika até a Semana Epidemiológica 13, 2016 [Internet]. Brasília: Ministério da Saúde; 2016 [acessado em 10 dez. 2018]. Disponível em: http://portalsaude.saude.gov.br/images/pdf/2016/ abril/27/2016-014---Dengue-SE13-substitui----o.pdf.

3. Burattini MN. Doenças infecciosas no Século XXI. Acta Paul Enferm [Internet]. 2016 [acessado em 10 dez. 2018];29(2):III-VI. Disponível em: http://dx.doi. org/10.1590/1982-0194201600018.

4. Lima AFC, Ortiz DR. Direct cost of development and documentation of the nursing process. Rev Bras Enferm [Internet]. 2015 [acessado em 1o dez. 2018];68(4):683-9. Disponível em: http://dx.doi. org/10.1590/0034-7167.2015680416i.

5. Doncliff B. Improving the quality of nursing notes. Nurs N Z [Internet]. 2015 [acessado em 3 dez. 2018];21(6):3-4. Disponível em: https://www.ncbi. nlm.nih.gov/pubmed/26402999.

6. Costa RK, Torres GV, Salvetti MG, Azevedo IC, Costa MA. Validity of instruments used in nursing care for people with skin lesions. Acta Paul Enferm [Internet]. 2014 [acessado em 5 dez. 2018];27(5):447-57. Disponível em: http://www. scielo.br/scielo.php?script $=$ sci $\operatorname{arttext} \&$ pid $=$ S010321002014000500010\&lng=en. http://dx.doi. org/10.1590/1982-0194201400074.

7. Marques DK, Souza GL, Silva AB, Silva AF, Nóbrega MM. International Nursing Minimum Data Set: a comparative study with tools of a pediatric clinic. Rev Bras Enferm [Internet]. 2014 [acessado em $10 \mathrm{dez}$. 2018];67(4):588-93. Disponível em: http://www. scielo.br/scielo.php?script $=$ sci $\operatorname{arttext} \&$ pid $=S 0034-$ 71672014000400588\&lng=en. http://dx.doi. org/10.1590/0034-7167.2014670414. 
8. Silva AF, Nóbrega MM, Souto CM. Instrument for documentation of nursing process during postpartum. Ciênc Cuid Saúde [Internet]. 2015 [acessado em 15 dez. 2018];14(3):1385-93. Disponível em: http:// periodicos.uem.br/ojs/index.php/CiencCuidSaude/ article/view/20227/15373. http://doi.org/10.4025/ cienccuidsaude.v14i3.20227.

9. Guimarães PV, Haddad MC, Martins EA. Instrument validation for assessing critically ill patients on mechanical ventilation according to the $\mathrm{ABCDE}$. Rev Eletr Enf [Internet]. 2015 [acessado em 14 dez. 2018];17(1):43-50. Disponível em: https://www.fen.ufg.br/fen revista/v17/n1/ pdf/v17n1a05.pdf. http://doi.org/10.5216/ree.v17i1.23178.

10. Burattini MN. Doenças infecciosas no Século XXI. Acta Paul Enferm [Internet]. 2016 [acessado em 15 dez. 2018];29(2):III-VI. Disponível em: http://www. scielo.br/scielo.php?script $=$ sci $\operatorname{arttext} \& \mathrm{pid}=\mathrm{S} 0103-$ 21002016000200001\&lng=pt. http://dx.doi. org/10.1590/1982-0194201600018.

11. Fehring R. Methods to validate nursing diagnosis. Heart Lung. 1987;16(6 Pt 1):625-9.

12. Hoskins LM. Clinical validation, methodologies for nursing diagnoses research. Int J Older People Nursing. 1989;8:309-18.

13. Lopes MVO, Silva VM, Araujo TL. Methods for establishing the accuracy of clinical indicators in predicting nursing diagnoses. Int J Nursing Knowledge [Internet]. 2012 [acessado em 20 dez. 2018];23(3):134-9. http://dx.doi.org/10.1111/j.2047-3095.2012.01213.x.

14. Horta WA. Processo de enfermagem. Rio de Janeiro: Guanabara Koogan; 2011.

15. Garcia TR, Cubas MC. Diagnósticos, intervençóes e resultados de enfermagem: subsídios para a sistematização da prática profissional. Rio de Janeiro: Elsevier; 2012.

16. Pasquali L. Instrumentação psicológica: fundamentos e práticas. Porto Alegre: Artmed; 2010.

17. Heale R, Twycross A. Validity and reliability in quantitative studies. Evid Based Nurs [Internet]. 2015 [acessado em $21 \mathrm{dez}$. 2018];18(3):66-7. Disponível em: http://dx.doi.org/10.1136/eb-2015-102129.

18. Carvalho RM, Guirado FSR, Neves WF, Rotta LA, Cotrim JB. Sepse por pneumonia estafilocócica adquirida na comunidade: relato de caso. Cuidarte Enferm [Internet]. 2016 [acessado em $21 \mathrm{dez} .2018$ ];10(2):18893. Disponível em: http://www.webfipa.net/facfipa/ ner/sumarios/cuidarte/2016v2/188-193.pdf
19. Aguirre S, Cuellar CM, Herrero Mb, Cortesi GC, Romero NG, Alvarez M, et al. Prevalence of tuberculosis respiratory symptoms and associated factors in the indigenous populations of Paraguay. Mem Inst Oswaldo Cruz [Internet]. 2017 [acessado em 21 dez. 2018];112(7):474-84. Disponível em: http://www. scielo.br/scielo.php?script $=$ sci $\operatorname{arttext} \&$ pid $=$ S007402762017000700474\&lng=en. http://dx.doi. org/10.1590/0074-02760160443.

20. Gudina EK, Gudissa FG. Prevalence of tuberculosis in HIV in Ethiopia in early HAART era: retrospective analysis. Pan Afr Med J [Internet]. 2013 [acessado em 22 dez. 2018];14:126. Disponível em: http://dx.doi. org/10.11604/pamj.2013.14.126.1907.

21. Santos AP, Navarro AM, Schwingel A, Alves TC, Abdalla PP, Venturini ACR, et al. Lipodystrophy diagnosis in people living with HIV/AIDS: prediction and validation of sex-specific anthropometric models. BMC Public Health [Internet]. 2018 [acessado em 10 jan. 2019];18:806. Disponível em: http://dx.doi. org/10.1186/s12889-018-5707-z.

22. Silva IR, Dias RM, Dutra CD, Mendes AN, Libonati RM. Dyslipidemia and nutritional status in patients HIV positive with syndrome lipodystrophy. Rev Epidemiol Control Infect [Internet]. 2014 [acessado em 22 dez. 2018];4(3):200-7. Disponível em: $\quad$ https://pdfs.semanticscholar.org/6a54/ ee1128ff4bc2c55285f39a351d0562d09692.pdf.

23. Santos LC, Lucinda LR, Silva GCR, Rocha ATG, Teixeira $\mathrm{R}$, Silva LD. Coexistência de ascite quilosa e trombose da veia porta: relato de caso e revisão da literatura. Rev Med Minas Gerais [Internet]. 2015 [acessado em 22 dez. 2018];25(1):129-32. Disponível em: http://www. dx.doi.org/10.5935/2238-3182.20150022.

24. Szerwieski LLD, Cortez LER, Marcon SS. O acompanhante do adulto hospitalizado na ótica da equipe de enfermagem. Rev Enferm UFPE [Internet]. 2016 [acessado em 7 abr. 2019];10(1):4856. Disponível em: http://www.dx.doi.org/10.5205/ reuol.8423-73529-1-RV1001201607.

25. Silva SK, Passos SMK, Souza LDM. Associação entre religiosidade e saúde mental em pacientes com HIV. Psicol Teor Prat [Internet]. 2015 [acessado em 7 abr. 2019];17(2):36-51. Disponível em: http://pepsic. bvsalud.org/scielo.php?script=sci arttext\&pid=S1516$\underline{36872015000200003 \& \operatorname{lng}=\text { pt\&nrm=iso. }}$ 